\title{
Bands, Bonds, and Polarizations in Nitrides - from Electronic Orbitals to Electronic Devices
}

\author{
J.A. Majewski, G. Zandler And P. VogL \\ Walter Schottky Institute, Technical University of Munich \\ Am Coulombwall 3, 85748 Garching, Germany
}

\begin{abstract}
A key property of the nitrides is the fact that they possess large spontaneous and piezoelectric polarization fields that allow a significant tailoring of the carrier dynamics and optical properties of nitride devices. In this paper, based on first-principles calculations of structural and electronic properties of bulk nitrides and their heterostructure, we investigate the potential of this novel material class for modern device applications by performing self-consistent Monte Carlo simulations. Our studies reveal that the nitride based electronic devices have characteristics that predispose them for high power and high frequency applications. We demonstrate also that transistor characteristics are favorably influenced by the internal polarization induced electric fields.
\end{abstract}

PACS numbers: 73.40.Kp, 77.65.Ly

\section{Introduction}

In recent years, huge progress in the III- $V$ nitride technology has been achieved. Commercial efforts have focused mostly on the optoelectronic applications (short wave LEDs and laser diodes) of nitrides but also nitride based electrical devices have attracted considerable interest. Such devices are promising candidates for high power, high frequency applications, which can range from cellular phones and satellite communications $(0.8-1.9 \mathrm{GHz})$ through direct broadcast satellite TV (11-19 GHz) to anticolliding radar $(80 \mathrm{GHz})$. Even already fabricated GaN transistors [1] exhibit breakdown voltages which are unreachable in devices based on other materials (see Fig. 1), however, the desirable range of $180-200 \mathrm{GHz}$ has not been reached yet. In the present paper, we review our recent studies of the nitride devices. We assess the potential of $\mathrm{GaN} / \mathrm{AlGaN}$ heterostructure field effect 


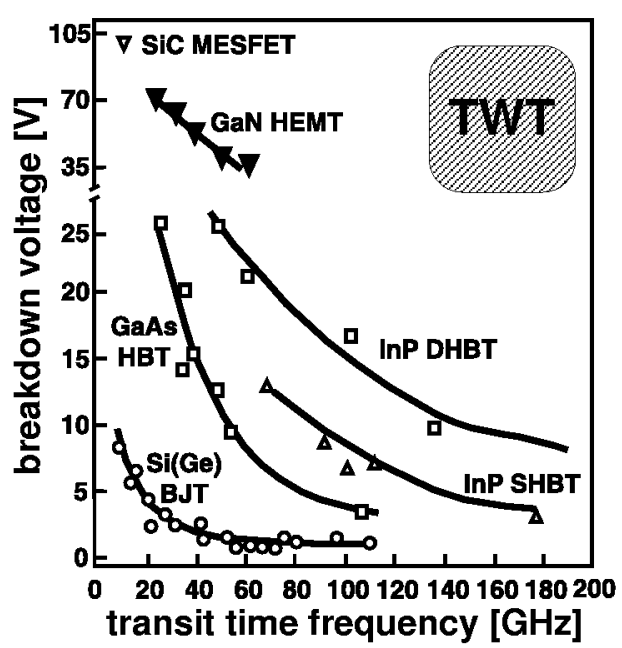

Fig. 1. Breakdown voltage and transit time frequency of electron devices based on various materials. The region covered by travelling wave tubes (TWT) is also marked.

transistors (HFETs) for very high speed electronics by performing self-consistent ensemble Monte Carlo simulations.

The strong internal electric fields in nitrides which result from their strong pyro- and piezoelectric constants [2] have a dramatic effect on the electronic and optical properties in these systems. In the GaN or InGaN quantum wells, the electric field in the well causes the red shift of transition energies and strongly suppresses the interband transitions [3]. This effect is generally considered as unfavorable for the optoelectronic devices based on multiple quantum wells [4]. On the other hand, the electric fields increase the electron and/or hole densities accumulated at the interfaces. This opens new possibilities for device engineering. In the present paper, we demonstrate how the pyro- and piezoelectric character of the nitrides can be utilized in the design of the novel HFETs. We show that the internal electric fields have a significant and favorable influence on the transistor characteristics.

The lack of detailed experimental data on transport parameters in nitride semiconductors is an obstacle in reliable device simulations. Therefore, we have performed extensive $a b$ initio electronic structure calculations which provide us the needed parameters. We have determined band offsets, the polarization induced charges at interfaces, the energies of all subsidiary conduction band minima relevant for high field transport, the effective masses of these valleys, and deformation potentials. The results of these studies will be reported shortly in Sec. 2. Since the two-dimensional (2D) electron gas is the most important component of the HFETs, the theoretical studies of its properties are described in Sec. 3. In Sec. 4, the results of transport Monte Carlo calculations are presented. Finally, the paper is summarized in Sec. 5 . 


\section{Theory of nitride bulks and their interfaces}

In this section, we discuss the influence of the polarization fields on properties of nitride heterostructures. The present systematic theoretical studies of structural and electronic properties of heteroepitaxial $\mathrm{AlN} / \mathrm{GaN}$ interfaces and homoepitaxial stacking faults [5], are based on a well-established first-principles total-energy pseudopotential method within the local-density-functional formalism [6]. The band offsets, the charge accumulation at the polar interfaces of the junctions, and the interface electronic states have been investigated, taking fully into account the effects of lattice relaxation and electric polarization. Before we turn to the nitride heterostructures let us describe first the detailed electronic structure of the conduction bands in bulk wurtzite GaN, which is essential for the description of carrier dynamics in nitride devices.

\subsection{Conduction bands in wurtzite $G a N$}

The wurtzite GaN exhibits a rich structure of the conduction band minima (Fig. 2). The relevant valley effective masses and the nonparabolicity parameters $\alpha$ in wurtzite GaN (wz-GaN) predicted by the first-principles calculations are given in Table. The conduction band minimum lies in the $\Gamma$-point. The second minimum lies in $U$ point, which is placed on the $M L$ line of the hexagonal Brillouin zone. We note that the sixfold degenerate $U$-point is equivalent to the three $X$ valleys and three out of four $L$ valleys of the zinc blende Brillouin zone. In wz-AIN, the minimum lies in $\Gamma$-point and the conduction band edge effective mass equals $0.32 m_{0}$.

\section{TABLE}

The lowest conduction band minima in wurtzite GaN, and their positions relative to the conduction band bottom (in eV), effective masses (in units of $m_{0}$ ), and anisotropy parameters.

\begin{tabular}{c|c|c|c|c}
\hline \hline Valley & $E($ valley $)-E\left(\Gamma_{c}\right)$ & $m_{\|}^{*}$ & $m_{\perp}^{*}$ & \multicolumn{1}{|c}{$\alpha$} \\
\hline$\Gamma_{c}$ & 0.0 & 0.18 & 0.20 & 0.6 \\
$U$ & 1.87 & 1.43 & 0.33 & 0.2 \\
$\Gamma_{c}^{3}$ & 2.08 & 2.17 & 0.28 & 0.3 \\
$M$ & 2.68 & 2.62 & 2.12 & 0.2 \\
$K$ & 2.77 & 0.65 & 0.40 & -0.4
\end{tabular}




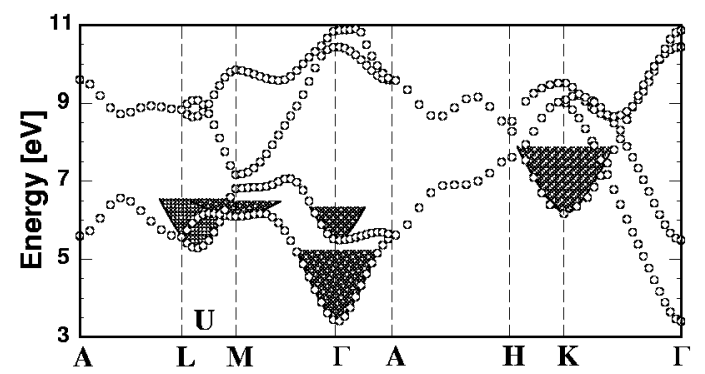

Fig. 2. Conduction bands of wurtzite GaN as obtained from density functional calculations.

\subsection{Internal polarization fields in quantum wells}

The space group $P 6_{3} m c\left(C_{6 v}^{4}\right)$ of wurtzite structure is compatible with a spontaneous polarization along the hexagonal $c$-axis. Therefore, the polarization can be of both pyroelectric and piezoelectric origin in wurtzite AlN and GaN, but only piezoelectric in the cubic phases. Whenever the polarization lies parallel to the growth direction, its change (divergence) across the interface is equivalent to an interface charge. Among the interfaces between GaN and AlN which we have studied, the [111] GaN/AlN, the [0001] GaN/AlN, and the stacking fault interface between the wurtzite and zinc blende $\mathrm{GaN}$ interface are of this type, which may be termed "polar". In cases such as [12 $\overline{1} 0] \mathrm{GaN} / \mathrm{AlN},[0 \overline{1} 10] \mathrm{GaN} / \mathrm{AlN}$, or [110] GaN/AlN, on the other hand, the polarization lies parallel to the interface and therefore does not give rise to a charge accumulation. We term those interfaces "nonpolar". A limiting case of this type is the cubic [001] GaN/AIN interface.

Most of the optoelectronic and electric nitride devices are based on the quantum wells. In such a structure, a thin layer of one semiconductor (e.g., GaN) constituting the well is embedded in another material (e.g., AlGaN). The vector of electric polarization in each material $(i=1,2)$ can be written as $\boldsymbol{P}^{(i)}=\boldsymbol{P}_{0}^{(i)}+\chi^{(i)} \boldsymbol{E}^{(i)}$, where zero field polarization $\boldsymbol{P}_{0}^{(i)}$ consists of spontaneous and/or piezoelectric polarization, and $\chi^{(i)}$ is susceptibility of material " $i$ ". The most interesting case is when the polarizations $\boldsymbol{P}_{0}^{(i)}$ are perpendicular to the interfaces (like in the [0001] GaN/AIN system, for example). Then the divergence of the polarizations in two semiconductors gives rise to the polarization charges at the interfaces $\sigma$ and further to the electric fields $\boldsymbol{E}^{(i)}$. The interface charge and electric field in the well are dependent on the boundary conditions imposed on the system. Here we consider three types of boundary conditions: (i) free standing system in vacuum, (ii) periodic boundary conditions, i.e., the potentials of the external surfaces of the cladding layers are equal, and (iii) neutralizing free charges $\sigma_{\mathrm{fL}}$ and $\sigma_{\mathrm{fR}}$ at the external surfaces. Further, we assume that the well has thickness $l_{1}$ and the 
two cladding layers on both sides of the well have thickness $l_{2}$ each. Using simple electrostatic arguments and considering the boundary conditions for the normal components of the electric displacements $D^{(i)}=4 \pi P_{0}^{(i)}+\epsilon_{i} E^{(i)}\left(\epsilon_{i}\right.$ - dielectric constant) at four interfaces (two external surfaces and two internal interfaces) for the cases (i)-(iii), one obtains the interface polarization charges $\pm \sigma$ and electric fields in the well $\left(E^{(1)}\right)$ and the cladding layers $\left(E^{(2)}\right)$ :

(i) $\sigma=\frac{P_{0}^{(2)}}{\epsilon_{2}}-\frac{P_{0}^{(1)}}{\epsilon_{1}}, \quad E^{(1)}=-\frac{4 \pi P_{0}^{(1)}}{\epsilon_{1}}, \quad E^{(2)}=-\frac{4 \pi P_{0}^{(2)}}{\epsilon_{2}}$,

(ii) $\sigma=\frac{\left(P_{0}^{(2)}-P_{0}^{(1)}\right)\left(2 l_{2}+l_{1}\right)}{2 l_{2} \epsilon_{1}+l_{1} \epsilon_{2}}, \quad E^{(1)}=4 \pi \sigma \frac{2 l_{2}}{2 l_{2}+l_{1}}, \quad E^{(2)}=-E^{(1)} \frac{l_{1}}{2 l_{2}}$,

(iii) $\sigma=\frac{P_{0}^{(2)}-P_{0}^{(1)}}{\epsilon_{1}}, \quad E^{(1)}=4 \pi \sigma, \quad E^{(2)}=0$.

Note that the relation $E^{(1)}-E^{(2)}=4 \pi \sigma$ holds in all three cases. In the case (iii), the free surface charges which cause the electric field in the cladding layer to vanish are $\sigma_{\mathrm{fL}}=-\sigma_{\mathrm{fR}}=P_{0}^{(2)}$. In the case (ii), the fields $E^{(1)}, E^{(2)}$, and the interface charge $\sigma$ tend to the corresponding quantities from the case (iii), if the cladding layer thickness increases to infinity $\left(l_{2} \rightarrow \infty\right)$. For semiconductors with very similar dielectric constants $\left(\epsilon_{1} \approx \epsilon_{2}\right)$, like in the case of AlGaN/GaN quantum wells, the interface polarization charges are independent of the boundary conditions at the external surfaces. Then, the quantum well with neutralized surface charges (i.e., $E^{(2)}=0$ ) can be well simulated in supercell calculations with periodic boundary conditions.

\subsection{GaN/AlN heterostructures}

In the following, we consider strained GaN layers which are grown pseudomorphically on cubic or wurtzite AIN substrate. For cubic heterostructures, we consider [001], [110], and [111], and for the wurtzite heterostructures [0001], [0110], and $[\overline{1} 2 \overline{1} 0]$ growth directions, respectively. In the case of stacking fault interfaces, we assume the growth axis to be the hexagonal $c$-axis ([0001] in wurtzite, [111] in zinc blende).

Computationally, we have modeled all of these interfaces by supercells con-

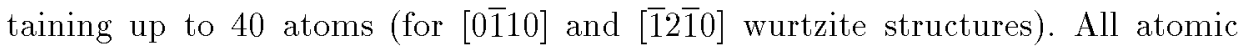
positions in the unit cell have been optimized by minimizing the total energy via the Hellmann-Feynman forces. The length of the supercell was determined by minimizing the stress tensor component along the growth direction. Based on these first-principles calculations, we find that the charges induced at the interfaces and valence band offsets (VBOs) are insensitive to the lattice constant along the growth direction, but highly sensitive to the atomic relaxation at the interface (e.g. the VBO for the relaxed [0001] GaN/AlN interface is $0.4 \mathrm{eV}$ smaller than for the unrelaxed one). The lattice relaxation near the interface reduces the interface charge approximately by a factor of two. 


\subsection{Valence band offsets and interface charges}

The calculated band lineups [7] of the studied heterostructures are depicted in Fig. 3. It is interesting to note that all calculated VBOs for AlN/GaN heterostructures are of the order of $0.7-0.9 \mathrm{eV}$ and are insensitive to the polar/ nonpolar character of the interface. Thus, the macroscopic electric fields do not grossly alter or modify the intrinsic band offsets. The presently predicted VBOs agree very well with recent experimental data [8,9], but not with some core-level photoemission data which suggest an extremely high VBO of $1.36 \mathrm{eV}$ [10]. The large difference between conduction band offsets in cubic and wurtzite GaN/AlN heterostructures is a consequence of the indirect energy gap $(\Gamma \rightarrow X)$ in cubic AlN which is $1.4 \mathrm{eV}$ smaller than the direct gap in wurtzite AlN. In GaN stacking fault (zb-GaN/wz-GaN) the VBO equals $0.04 \mathrm{eV}$, whereas in AlN stacking fault (not shown in Fig. 3) the VBO is equal to $0.02 \mathrm{eV}$. All studied GaN/AlN interfaces and stacking faults are of type I, with the valence band lying higher and the conduction band being lower in $\mathrm{GaN}$, and zinc blende phase of the stacking fault, respectively.

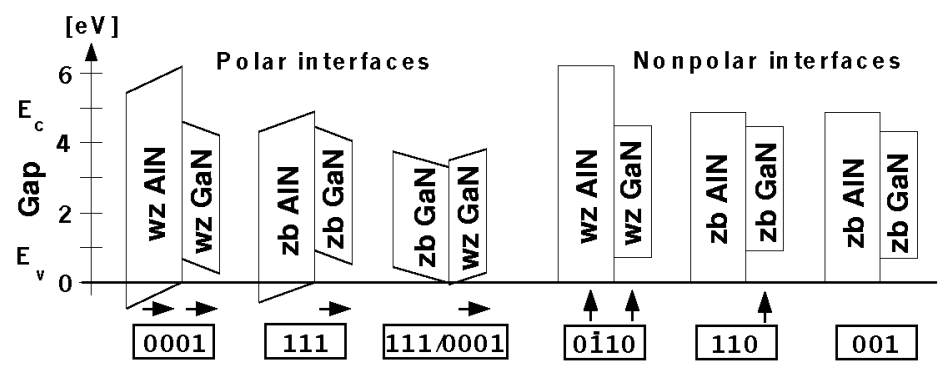

Fig. 3. Predicted band lineups for several AlN/GaN interfaces. The growth direction is given in boxes beneath each interface. The arrows indicate the direction of the spontaneous or strain-induced piezoelectric polarization. Polar interfaces possess an interface charge. The corresponding electric field induces a band bending which is indicated schematically.

The calculated charges at the polar interfaces are equal to $0.010 \mathrm{C} / \mathrm{m}^{2}$ and $0.006 \mathrm{C} / \mathrm{m}^{2}$ for [0001] wz-AlN/wz-GaN and [111] zb-AlN/zb-GaN heterostructures, respectively. The large charges accumulated at the stacking fault interfaces (i.e., 0.009 and $0.003 \mathrm{C} / \mathrm{m}^{2}$ for $\mathrm{AlN}$ and $\mathrm{GaN}$ stacking faults, respectively) result mainly from the spontaneous dielectric polarization of the hexagonal phase, whereas the strain-induced piezoelectric contribution is negligible. The presently calculated charges compare well (the difference amounts to 15-20\%) with results obtained from electrostatic arguments (Eqs. (2) and (3)), in which the bulk values of the spontaneous polarization, and piezoelectric constants [2] have been used. The stacking faults can generate a persistent photoconductivity and they can be 
tuned to act either as excitonic traps or luminescence centers, depending on the width of the cubic layer.

Our calculations reveal the existence of large internal electric fields. Their influence on the properties of the electronic devices will be presented in subsequent sections.

\section{2DEG in AlGaN/GaN single heterostructures}

As discussed in Sec. 2, the electric polarization in nitride structures induces the polarization charge at the heterostructure interface. The two-dimensional electron gas (2DEG) emerges at the interface with positive polarization charge. To study the transport properties of the $2 \mathrm{DEG}$, we focus on a single $\mathrm{Al}_{0.15} \mathrm{Ga}_{0.85} \mathrm{~N} /$ GaN heterostructure (see Fig. 4) first. The heterostructure consists of a tensile strained $30 \mathrm{~nm}$ thick $\mathrm{Al}_{0.15} \mathrm{Ga}_{0.85} \mathrm{~N}$ and a $\mathrm{Ga}$-face (i.e., with spontaneous polarization towards the substrate) the relaxed $300 \mathrm{~nm}$ thick GaN layer. In AlGaN layer, there is polarization of both pyro- and piezoelectric origins, while the GaN layer contains the pyroelectric moment only. We assume that there are some compensating charges at the surface and the nucleation layer. The polarization charge at the $\mathrm{Al}_{0.15} \mathrm{Ga}_{0.85} \mathrm{~N} / \mathrm{GaN}$ interface has been taken to be $6 \times 10^{12} \mathrm{~cm}^{-2}$, which interpolates between a recent experimental value [11] $4 \times 10^{12} \mathrm{~cm}^{-2}$ and the theoretical value $8 \times 10^{12} \mathrm{~cm}^{-2}$ calculated for full compensation. Figure 4 depicts the self-consistent band edge and density profiles along the growth direction obtained from the coupled 1-dimensional Schrödinger and Poisson equations. The Poisson
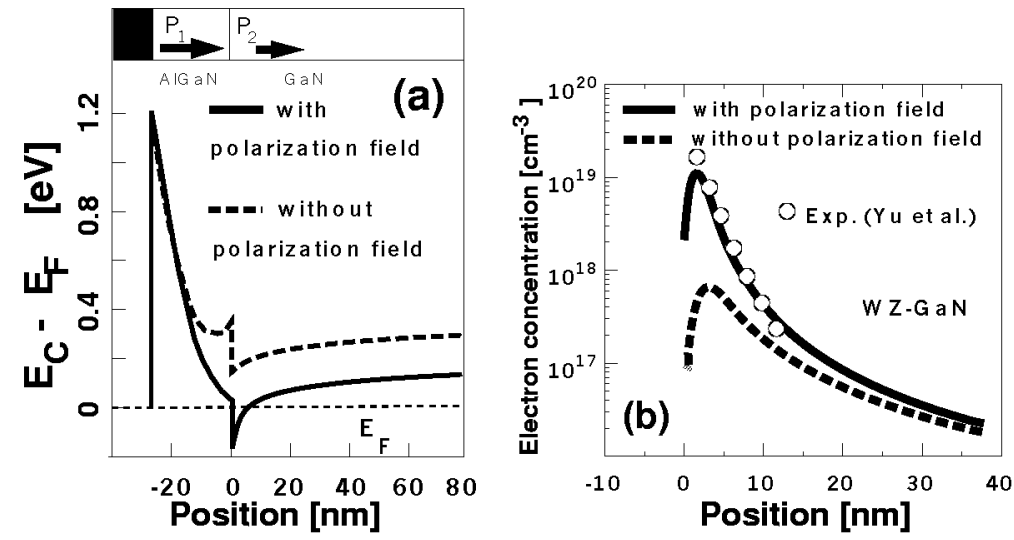

Fig. 4. Calculated results for a $\mathrm{Al}_{0.15} \mathrm{Ga}_{0.85} \mathrm{~N} / \mathrm{GaN}$ heterostructure with (full lines) and without (dashed lines) spontaneous and piezoelectric fields. A homogeneous $n$-doping background of $10^{16} \mathrm{~cm}^{-3}$ throughout the structure has been assumed. (a) Self-consistently computed conduction band edge profile along the growth direction.

(b) Calculated electron density in comparison with published experimental values. 
equation includes all interface charges which result from the polarization fields. As shown in Fig. 4, the polarization induced positive interface charge causes a stronger confinement of $2 \mathrm{D}$ electron channel and an increase in the channel density by more than an order of magnitude. This is consistent with the experimental values for this layer structure [11].

Furthermore, we have performed ensemble Monte Carlo calculations for drift mobilities along the channel which forms parallel to the interface. The inter- and intrasubband-scattering rates [12] have been determined consistently with the confined electronic channel states. We find excellent agreement between experimental and calculated temperature dependent mobilities [12], consistent with the high channel densities reported for these devices $[11,13]$. We would like to stress that such type of agreement strongly supports the existence of the strong pyroelectric polarization fields. For channel densities exceeding $10^{13} \mathrm{~cm}^{-2}$, we predict a phonon limited drift mobility for $2 \mathrm{D}$ electrons close to $2000 \mathrm{~cm}^{2} /(\mathrm{V} \mathrm{s})$ for high quality interfaces, in good agreement with recent data on such structures [14].

\section{Monte Carlo simulations of nitride devices}

Before we turn to the heterostructure based transistors, let us shortly discuss the transport properties of bulk nitrides.

\subsection{Bulk transport properties of $G a N$ and $A l N$}

In our Monte Carlo transport calculations, we have used band parameters deduced from the electronic structure calculations described above and have taken into account scattering rates for ionized impurity, intra- and intervalley phonon scattering, acoustic, piezoelectric, polar optical phonon and alloy scattering. The resulting electron drift velocities (see Fig. 5) at room temperature in bulk GaN

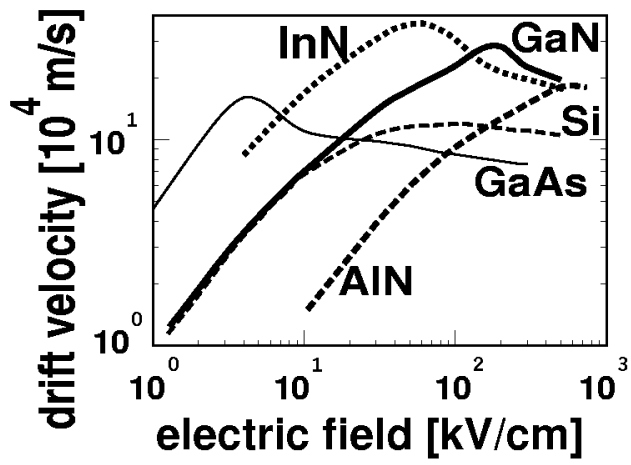

Fig. 5. Calculated electron drift velocities of $\mathrm{GaN}, \mathrm{AIN}$, and $\mathrm{InN}$ in comparison with Ga.As and Si. 
both at low and high fields are in good agreement with other published theoretical results [15]. At high fields, GaN, AlN as well as InN show a saturation velocity $\left(\approx 1.5 \times 10^{7} \mathrm{~cm} / \mathrm{s}\right.$ at $\left.1 \mathrm{MV} / \mathrm{cm}\right)$ which is twice as high as in GaAs. For AlN, the heavier effective mass leads to considerably lower drift velocities at low fields. These results indicate that one can achieve much higher transit time frequencies in submicron nitride devices than in GaAs ones.

\section{D. Characteristics of AlGaN/GaN HFETs}

To study the influence of polarization fields on device characteristics, we have performed self-consistent two-dimensional Monte Carlo simulations of various nitride based heterostructure devices. The results for HFET based on InGaN quantum well have been reported previously [7, 16]. It has been found that the polarization field pushes the electrons closer to the gate contact and therefore increases the transconductance. Furthermore, this confining effect improves the turn off behavior of the device considerably.

Here, we present results of Monte Carlo simulations for the standard design HFETs consisting of the slightly $p$-type doped GaN film and $25 \mathrm{~nm}$ wide undoped barrier of $\mathrm{Al}_{0.30} \mathrm{Ga}_{0.70} \mathrm{~N}$. These HFETs, owing to the strong spontaneous and piezoelectric polarizations, yield high density of $2 \mathrm{D}$ electron gas $\left(\approx 10^{13} \mathrm{~cm}^{-2}\right)$ without intentional doping. It turns out to be favorable, since initial doping decreases electron mobility and increases gate tunnel leakage [1]. We consider the transistors with gate length ranging from 150 to $300 \mathrm{~nm}$. The various source-gate $(275-600 \mathrm{~nm})$ and gate-drain $(475-1200 \mathrm{~nm})$ distances used in the simulations correspond to the parameters of experimental structures. The polarization induced interface charge has been estimated to be $1.6 \times 10^{13} \mathrm{~cm}^{-2}$.

The current-voltage characteristics for transistor with gate lengths of 150 and $300 \mathrm{~nm}$ are depicted in Fig. 6. The drain current is very large and increases only moderately with decreasing gate length. Further, we find out that the increase
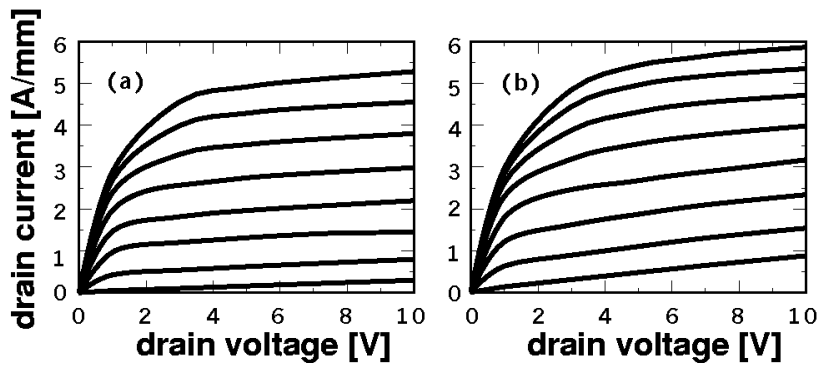

Fig. 6. Drain current characteristics for gate lengths (a) $300 \mathrm{~nm}$ and (b) $150 \mathrm{~nm}$, as obtained from Monte Carlo simulations for $\mathrm{Al}_{0.30} \mathrm{Ga}_{0.70} \mathrm{~N} / \mathrm{GaN}$ HFET. The various gate voltages are given in steps of $1 \mathrm{~V}$ and begin with $-8.4 \mathrm{eV}$ (the lowest line). 
in source-gate spacing decreases the drain current, whereas, increased gate-drain distance influences the current only in the region of low voltage $V$.

The simulations predict the maximum transconductance to be roughly $900 \mathrm{~S} / \mathrm{m}$ (see Fig. 7), which is a factor of two larger than the transconductance of currently realized transistors. The results for transit time calculated from average channel velocity are presented in Fig. 8. The present Monte Carlo calculations predict average channel velocities exceeding $2 \times 10^{5} \mathrm{~m} / \mathrm{s}$ for gate lengths below $200 \mathrm{~nm}$. The transit time scales almost with the inverse of the effective gate length and can reach $250 \mathrm{GHz}$ in the systems studied. It is much larger than extrinsic transit times measured in experimental structures $(\approx 40 \mathrm{GHz})$. Very similar characteristics have been also calculated for the HFET with $\mathrm{Al}_{0.83} \operatorname{In}_{0.17} \mathrm{~N}$ quantum well and identical geometry parameters as in the HFET described above. In conclusion, we predict excellent high frequency and power performance for wide-gap nitride based HFETs with gate lengths of the order of $200 \mathrm{~nm}$.

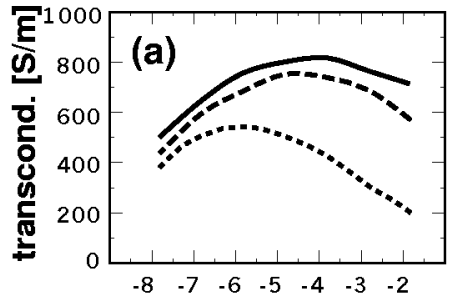

gate potential [V]

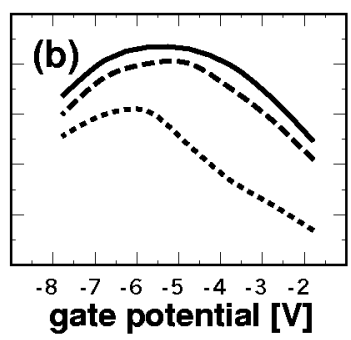

Fig. 7. Calculated transconductance for $\mathrm{Al}_{0.30} \mathrm{Ga}_{0.70} \mathrm{~N} / \mathrm{GaN}$ HFET with two different gate lengths (a) $300 \mathrm{~nm}$, (b) $150 \mathrm{~nm}$ for three gate voltages equal to $1 \mathrm{~V}$ (dotted line), $3 \mathrm{~V}$ (dashed line), and $10 \mathrm{~V}$ (continuous line).
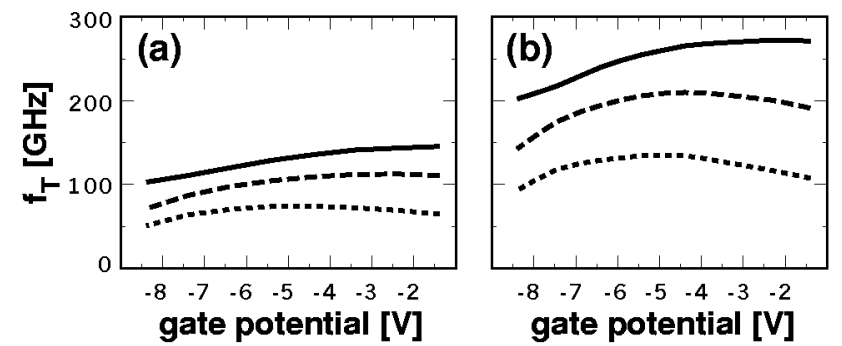

Fig. 8. Calculated transit time frequencies for $\mathrm{Al}_{0.30} \mathrm{Ga}_{0.70} \mathrm{~N} / \mathrm{GaN}$ HFET with two different gate lengths (a) $300 \mathrm{~nm}$, (b) $150 \mathrm{~nm}$ for three gate voltages equal to $1 \mathrm{~V}$ (dotted line), $3 \mathrm{~V}$ (dashed line), and $10 \mathrm{~V}$ (continuous line).

\subsection{Theoretically designed inverted HFET}

Polarization induced internal fields can be effectively utilized to tailor devices with optimal potential barriers towards the substrate. Indeed, we have theoretically designed the following inverted HFET structure which should yield high 
channel densities and an excellent turn-off behavior. On top of a relaxed GaN buffer, there is a $12 \mathrm{~nm}$ thick strained $\mathrm{Al}_{0.40} \mathrm{Ga}_{0.60} \mathrm{~N}$ layer. The inner part $(8 \mathrm{~nm})$ of this layer is highly $n$-doped with $1.9 \times 10^{19} \mathrm{~cm}^{-3}$. The remaining part of the barrier is moderately doped with $10^{17} \mathrm{~cm}^{-3}$ carriers. On top of this layer, there is a $30 \mathrm{~nm} \mathrm{GaN} \mathrm{(} n$-doped with $4 \times 10^{16} \mathrm{~cm}^{-3}$ ). The gate is assumed to be recessed in order to achieve a small gate to channel separation of $14 \mathrm{~nm}$. In this HFET, we assume the opposite polarity of the structure as in the previous example, i.e., we have N-face GaN film with spontaneous polarization directed outward the substrate. The GaN is assumed to be field free, whereas two GaN/AlGaN interfaces have opposite interface charges equal to $\pm 2 \times 10^{13} \mathrm{~cm}^{-2}$. The calculated band edge profile, together with the confined electron density is shown in Fig. 9a at midgate position.
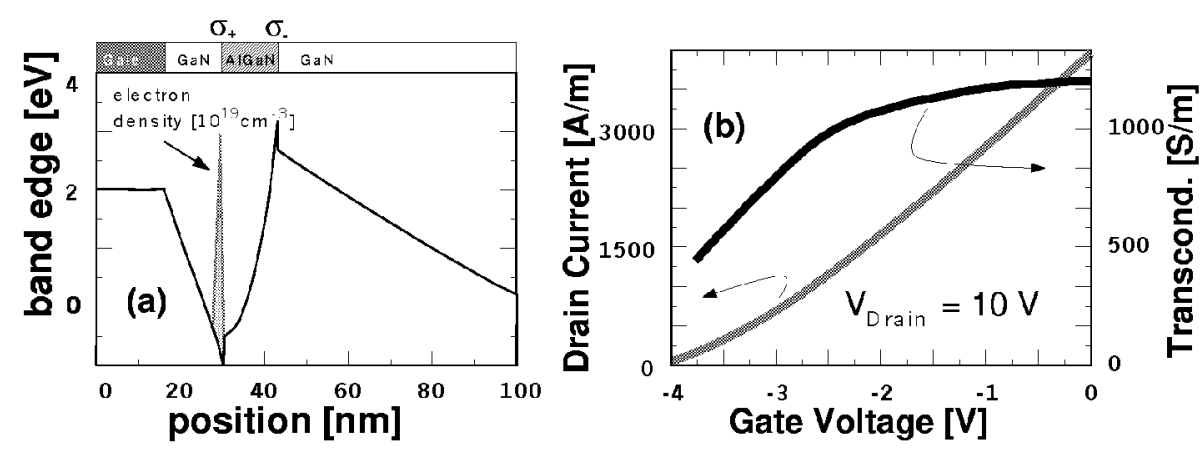

Fig. 9. Monte Carlo results for a $300 \mathrm{~nm}$ gate length inverted $\mathrm{GaN} / \mathrm{Al}_{0.40} \mathrm{Ga}_{0.60} \mathrm{~N}$ HFET. (a) Self-consistent band edge profile for $-1 \mathrm{~V}$ gate voltage and $10 \mathrm{~V}$ drain voltage. The channel electron density is indicated schematically. (b) Drain current and transconductance versus gate voltage at $10 \mathrm{~V}$ drain voltage.

As can be seen from Fig. 9, the polarization induced field produces a high potential barrier of approximately $3 \mathrm{eV}$ towards the substrate. This has two major consequences. First, the highly doped AlGaN barrier gets completely depleted and all electrons are strongly confined in the channel which forms at the interface with the top GaN layer. Secondly, the high barrier completely suppresses the leakage current across the AlGaN barriers up to high voltages. We predict excellent electrical characteristics for such types of devices. In the present example, we find drain currents exceeding $3000 \mathrm{~A} / \mathrm{m}$ and a transconductance which lies well above $1000 \mathrm{~S} / \mathrm{m}$. We note that the thickness of the AlGaN barrier layer and its doping level must be chosen carefully in order to avoid the formation of parasitic hole channels.

\section{Conclusions}

In conclusion, pyro- and piezoelectrical polarizations must be taken into account in order to explain experimentally observed mobilities and densities in nitride 
heterostructures. They are crucial for device performances and offer a unique possibility to design novel high power, high frequency heterostructure transistors. The nitride based HFETs have potential to substitute the travelling wave tubes which are commonly used nowadays for high power and high frequency applications.

\section{Acknowledgments}

The authors kindly acknowledge fruitful discussions with Oliver Ambacher and Vincenzo Fiorentini. This work has been supported by the Deutsche Forschungs gemeinschaft (Project SFB 348).

\section{References}

[1] L.F. Eastman, V. Tilak, J. Smart, B.M. Green, E.M. Chumbes, R. Dimitrov, H. Kim, O.S. Ambacher, N. Weimann, T. Prunty, M. Murphy, W.J. Schaff, J.R. Shealy, IEEE Trans. Electron Devices 48, 479 (2001).

[2] F. Bernardini, V. Fiorentini, D. Vanderbilt, Phys. Rev. B 56, 10024 (1997).

[3] M. Leroux, N. Grandjean, M. Laügt, J. Massies, B. Gil, P. Lefebvre, P. Bigenwald, Phys. Rev. B 58, 13371 (1998).

[4] M.B. Nardelli, K. Rapcewicz, J. Bernholc, Appl. Phys. Lett. 71, 3135 (1997).

[5] X.H. Wu, L.M. Brown, D. Kapolnek, S. Keller, B. Keller, S.P. DenBaars, J.S. Speck, J. Appl. Phys. 80, 3228 (1996).

[6] M. Städele, J.A. Majewski, P. Vogl, Phys. Rev. B 56, 6911 (1997); J.A. Majewski, M. Städele, P. Vogl, MRS Internet J. Nitride Semicond. Res. 1, 30 (1996).

[7] G. Zandler, J.A. Majewski, P. Vogl, J. Vac. Sci. Technol. B 17, 1617 (1999).

[8] G. Martin, A. Botchkarev, A. Rockett, H. Morkoc, Appl. Phys. Lett. 68, 2541 (1996).

[9] G. Martin, S. Strite, A. Botchkarev, A. Agarwal, A. Rockett, H. Morkoç, W.R.L. Lambrecht, B. Segall, Appl. Phys. Lett. 65, 610 (1994).

[10] J.R. Waldrop, R.W. Grant, Appl. Phys. Lett. 68, 2879 (1996).

[11] E.T. Yu, G.J. Sullivan, P.M. Asbeck, C.D. Wang, D. Qiao, S.S. Lau, Appl. Phys. Lett. 71, 2794 (1997).

[12] R. Oberhuber, G. Zandler, P. Vogl, Appl. Phys. Lett. 73, 818 (1998).

[13] Y.F. Wu, B.P. Keller, D. Kapolnek, P. Kozodoy, S.P. DenBaars, U.K. Mishra, Appl. Phys. Lett. 69, 1438 (1996).

[14] R. Gaska, J.W. Yang, A. Osinsky, Q. Chen, M.A. Khan, A.O. Orlov, G.L. Snider, M.S. Shur, Appl. Phys. Lett.72, 707 (1998).

[15] U.V. Bhapkar, M.S. Shur, J. Appl. Phys. 82, 1649 (1997); S.K. O’Leary, B.E. Foutz, M.S. Shur, U.V. Bhapkar, L.F. Eastman, Solid State Commun. 105, 621 (1988).

[16] G. Zandler, J.A. Majewski, M. Städele, P. Vogl, F. Compagnone, Phys. Status Solidi B 204, 133 (1997). 